\title{
Value of monoclonal anti-myeloperoxidase (MPO7) for diagnosing acute leukaemia
}

\author{
J Storr, G Dolan, E Coustan-Smith, D Barnett, J T Reilly
}

\begin{abstract}
The expression of myeloperoxidase (MPO) was studied in 100 cases of acute leukaemia ( 83 with acute myeloid leukaemia (AML) and 17 acute lymphoblastic leukaemia (ALL)) by both a conventional cytochemical method and the immunocytochemical antiperoxidase (APAAP) technique using the monoclonal antibody MPO7. In each case the staining was evaluated by light microscopical examination (percentage of positive cells). Of the 83 cases of AML, 78 (93.9\%) were positive for MPO7 compared with $70(84 \cdot 3 \%)$ by cytochemistry. Antibodies against the myeloid markers CD13 and CD33 were positive in 71 $(85 \cdot 5 \%)$ and $70(84 \cdot 3 \%)$ cases, respectively. Importantly, all cases of ALL were negative for both MPO7 and cytochemical MPO staining even when they were positive for $\mathrm{CD} 13$ and $\mathrm{CD} 33$. These results indicate that the antimyeloperoxidase antibody MPO7 is the most sensitive and specific reagent for the diagnosis of AML and should therefore be included in routine immunophenotyping panels.
\end{abstract}

Accurate classification of acute leukaemia, particularly the distinction between acute myeloid leukaemia (AML) and acute lymphoblastic leukaemia (ALL), has important clinical consequences. The usefulness of immunophenotyping is well known in the diagnosis of ALL. Cytology and cytochemistry are mostly used in the classification of $\mathrm{AML}$, and form the basis of classification in the French-American-British (FAB) system. ${ }^{1}$ The main limitations of monoclonal antibodies against myeloid antigens are that they may react with only a proportion of cases ${ }^{2}$ and some have been shown to lack specificity for AML. ${ }^{23}$

Myeloperoxidase (MPO) is a lysosomal enzyme found in the primary granules of cells of the myeloid series. ${ }^{4}$ The expression of MPO occurs early in myeloid differentiation and seems to be specific for cells of this lineage. ${ }^{5}$ Importantly, MPO has never been reported in ALL even in those rare cases expressing MPO mRNA. ${ }^{6}$

Conventional cytochemistry is widely used for the demonstration of "active" enzyme in leukaemic cells, but very low concentrations of MPO antigen can now be detected using monoclonal antibodies and the alkaline phos- phatase-antialkaline phosphatase (APAAP) technique. $^{7}$

This study compares the sensitivity and specificity of monoclonal anti-MPO (MPO7) with that of other monoclonal antibodies as well as with conventional cytochemical staining.

\section{Methods}

Peripheral blood and bone marrow samples were obtained from patients with acute leukaemia. A haematological diagnosis was based on Romanowsky staining, cytochemistry, and immunophenotyping. The panel of monoclonal antibodies against surface antigens included CD13, CD33 (Coulter/Seralab) CD14, CD19, anti-HLA-DR and CD7 (Becton-Dickinson/Royal Free Hospital). Analysis was performed by flow cytometry (Facsan, Becton-Dickinson), and the data analysed using a Consort 30 program. Cytoplasmic CD3 (Becton-Dickinson/Seralab), CD22 and $\mathrm{U}$ (Sera lab), and TdT (Dakopatts A/S) expression were assessed by indirect immunofluorescence. Samples were regarded as positive if an antigen was expressed by more than $10^{\circ}{ }_{0}$ of cells.

MPO was assessed in each case using two techniques, a standard cytochemical technique using the Hanker-Yates method ${ }^{89}$ and an alkaline phosphatase anti-alkaline phosphatase APAAP technique, ${ }^{7}$ using the monoclonal antibody MPO7 (obtained from Dakopatts A/S). Samples were regarded as positive if more than $3^{\circ}{ }_{0}$ of blasts stained with cytochemical $\mathrm{MPO}^{6}$ and greater than $10 \%$ with MPO7.

Cases of AML were subdivided according to the FAB classification and results of cytochemical and immunocytochemical investigations compared.

\section{Results}

The 100 cases of acute leukaemia were classified as AML ( $n=83)$ and ALL $(n=17)$ by standard FAB criteria. ${ }^{1}$ Of the cases of AML, $78(93.8 \%)$ were positive for MPO7 compared with $70(84.3 \%)$ for cytochemistry. Antibodies against the myeloid antigens CD13 and CD33 were positive in $71(85.5 \%)$ and 70 $(84.3 \%)$ of cases, respectively. The percentage of leukaemic cells reacting with MPO7, CD13, CD33 and cytochemical MPO for each FAB group are shown in fig 1 .

Five $(6 \%)$ cases of AML were negative for MPO7 while $13(15.6 \%)$ cases were negative 
Figure 1 The percentage of leukaemic cells positive by $(A) M P O 7^{\star},(B)$

cytochemical MPO, (C)

$C D 13(1 / 5)(D) C D 33$ $(1 / 5)$.

^MPO7 positivity in the case of the M7 AML was restricted to myeloblast subpopulation.
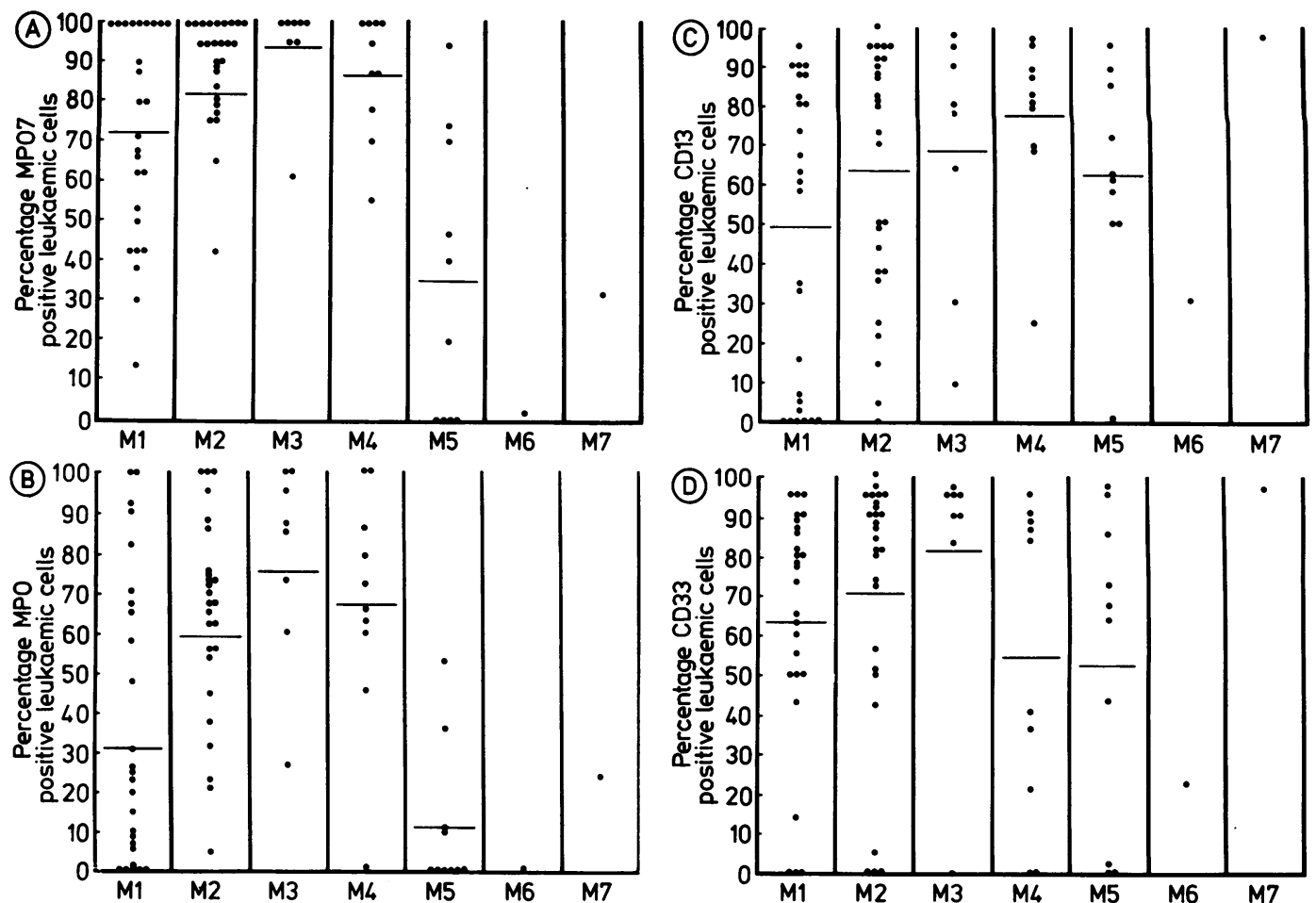

by the cytochemical MPO method; 12 $\left(14.4^{\circ}{ }_{0}\right)$ and $13\left(15.6^{\circ}\right)$ cases were negative for $\mathrm{CD} 13$ and $\mathrm{CD} 33$, respectively. All cases negative for MPO7 were also negative by cytochemistry. Of the four cases of M5 from this group, two were positive for both $\mathrm{CD} 13$ and $\mathrm{CD} 33$, while the remaining two were positive for only one antigen. These four cases, however, were positive for the monocytoid determinant CD14. The M6 case negative for MPO7 was positive for both CD13 and CD33. Cases failing to react with MPO7 seemed to be mainly confined to the M5 subtype while those cases negative for cytochemical MPO, CD13, and CD33 were independent of subtype (fig 1 ).

Results of immunological and cytochemical techniques for myeloperoxidase detection are shown in fig 2 . While both are relatively simple, the percentage cell positivity was consistently greater using MPO7 (fig 3). Interestingly, Auer rods were less easily shown by MPO7 than by cytochemistry, probably

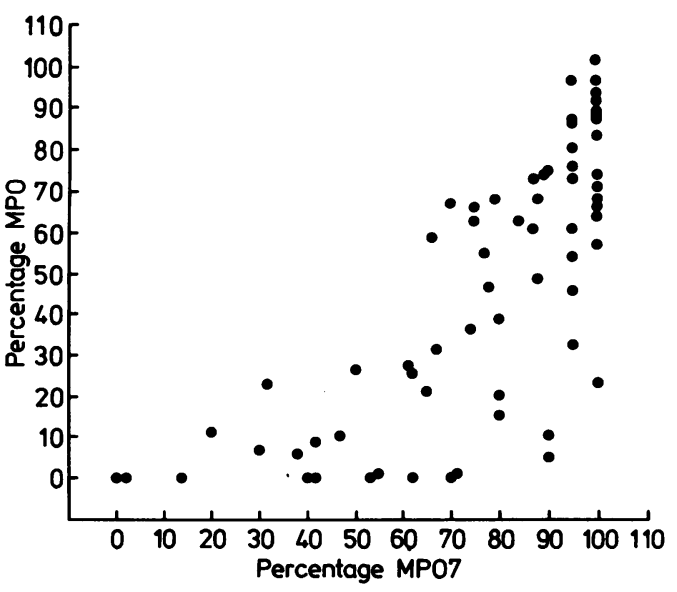

Figure 3 A comparison of the percentage of cells positive in each case using both the MPO7 and cytochemical techniques $(r=0 \cdot 781)$.
Figure 2 (A) Leukaemic cells stained using the APAAP technique; MPO7 diluted $1 / 50$.

Figure 2 (B) Leukaemic cells stained for myeloperoxidase using the cytochemical technique. 
because they were obscured by the denser staining achieved with this technique.

All 17 cases of ALL were negative with MPO7 and cytochemical MPO while 10 and eight cases exhibited CD13 and CD33 positivity, respectively. Six cases were positive for both CD13 and CD33.

\section{Discussion}

The accurate diagnosis of AML is important for appropriate treatment and management. Morphology and cytochemistry are not sufficiently precise to enable all cases of AML to be distinguished from those of ALL. MPO detection is probably the most specific technique for differentiating myeloid from lymphoid lineages. ${ }^{10}$

Our results show that MPO7 is the most sensitive method for distinguishing AML, with only $6^{\circ}$ o of cases negative. Of these, four were subtyped as M5 on the basis of reactivity with anti-CD14, and one as M6 by glycophorin positivity. Therefore, the combined use of only two antibodies-namely, MPO7 and CD14-successfully characterised $99 \%$ of cases of AML. Importantly, all cases belonging to subgroup $M 1$ reacted positively with MPO7. This is important as these cases are the most difficult to differentiate from ALL, not only on morphological grounds, but also because they most frequently coexpress lymphoid antigens. ${ }^{11}$ Cytochemistry and standard myeloid antibodies were less sensitive in the diagnosis of $M 1$, with six $(23 \%)$ negative by cytochemistry, nine $(39 \%)$ negative using anti-CD 13, and three $(13 \%)$ negative with anti-CD33. The discrepancy between immunological and cytochemical MPO detection is probably due to the presence of an antigenically intact but non-functioning enzyme, such as proMPO, in these cells. This has been reported to occur in the myeloblastic cell line KG-1. ${ }^{12}$

All cases of ALL were negative for myeloperoxidase both by MPO7 and cytochemistry, confirming the enzyme specificity, though this observation was based on a relatively small number of cases and should be confirmed using larger numbers. This degree of specificity was not found with other "myeloid" antibodies as, for example, over half the cases of ALL reacted with anti-CD13. The greater incidence of "lineage promiscuity" in this study, compared with that reported previously, ${ }^{13}$ may reflect the small number of cases of ALL studied and the fact that both centres receive a disproportionate number of diagnostically difficult cases. It is not explained by our definition of positivity as the results are not significantly changed if this is increased from $10 \%$ to $20 \%$ or greater.

These results confirm those of a recently published study which used an indirect immunofluorescence technique and an inhouse anti-MPO. ${ }^{14}$ We suggest, therefore, that MPO7, in conjunction with the APAAP technique, which permits individual cell identification, is more sensitive than alternative anti-myeloid antibodies and standard cytochemistry. ${ }^{15}$

This work was supported in part by the Haematology Research Fund (Northern General Hospital). We also thank Dakopatts A/S for their help with this project. We are also indebted to Dr D Campana for helpful discussion and advice.

1 Bennett JM, Catovsky D, Daniel MT, et al. Proposals for the classification of the acute leukaemias: FrenchAmerican-British (FAB) co-operative group. $\mathrm{Br} J$ Haematol 1976;33:451-8.

2 Drexler HG. Classification of acute myeloid leukaemias-A comparison of FAB and immunophenotyping. Leukaemia 1987;1:697-705.

3 Bradstock KF, Kirk J, Grimsley PG, Kabral A, Hughts WG. Unusual immunophenotyes in acute leukaemias: incidence and clinical correlations. Br J Haematol 1989;72:512-8.

4 Bainton DF, Ullyot JL, Farquhar MG. The development of neutrophilic polymorphonuclear leucocytes in human bone marrow: origin and content of azurophil and specific granules. J Exp Med 1971;134:907-34.

5 Moreshita Y, Moreshima Y, Ogura M, Nagai Y, Ohno R. Biochemical characterization of human myeloperoxidase using three specific monoclonal antibodies. Br J Haematol using three specific

6 Ferrari S, Mariano MT, Tagliafico E, et al. Myeloperoxidase gene expression in blast cells with a lymphoid phenotype in cases of acute lymphoblastic leukaemia. Blood 1988;72:873-6.

7 Davey FR, Erber WN, Gatter KC, Mason DY. Immunophenotyping of acute myeloid leukaemia by immuno-alkaline phosphatase (APAAP) labelling with a panel of antibodies. Am J Hematol 1987;26:157-66.

8 Hanker JS, Ambrose WW, James CJ, et al. Facilitated light microscopic cytochemical diagnosis of acute myelogenous leukaemia. Cancer Res 1979;39:1635.

9 Laycock BJ, Britton JA, Lilleyman JS. A technique avoiding carcinogens for the demonstration of myeloperoxidase in blood and bone marrow smears. J Clin Pathol 1980;33:194-6.

10 Chang KS, Trujillo JM, Cook RG, Stass SA. Human myeloperoxidase gene: Molecular cloning and expression in leukaemic cells. Blood 1986;68:1411-4

11 Jani P, Verby W, Greaves MF, Bevan D, Bollum FJ. Terminal deoxynucleotidyl transferase in acute myeloid leukaemia. Leuk Res 1983;7:17-29.

12 Koeffler HP, Ranyard J, Pertcheck M. Myeloperoxidase its structure and expression during myeloid differentiation. Blood 1985;65:484-91.

13 Sobol RE, Mick MS, Royston I, et al. Clinical importance of myeloid antigen expression in adult acute lymphoblastic meeloid antigen expression in adult acute ly

14 van der Schoot C, Daams GM, Pinkster J, Vet R, von dem Borne AEG Kr. Monoclonal antibodies against myeloperoxidase are valuable immunological reagents for the diagnosis of acute myeloid leukaemia. Br J Haematol 1990;74:173-8.

15 de Oliveira MSP, Matutes E, Rani S, Morilla R, Catovsky D. Early expression of MC52 (CD13) in the Cytoplasms of blast cells from Acute Myeloid Leukaemia. Acta Haematol 1988;80:61-4. 